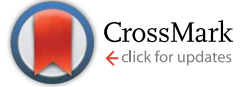

Cite this: RSC Adv., 2017, 7, 14504

Received 10th January 2017

Accepted 27th February 2017

DOI: 10.1039/c7ra00372b

rsc.li/rsc-advances

\section{Super water absorbency OMMT/PAA hydrogel materials with excellent mechanical properties}

\author{
Yuqi Zhang, ${ }^{a}$ Yao Liu, ${ }^{a}$ Jiaqi Liu, ${ }^{a}$ Pu Guo a and Liping Heng ${ }^{\star b}$
}

Superabsorbent hydrogels with excellent mechanical properties are fabricated by introducing organic montmorillonite (OMMT) into poly(acrylic acid) (PAA) hydrogels via simple UV irradiation polymerization. The obtained OMMT/PAA composite hydrogels are composed of a three-dimensional macroporous network structure. The effects of deionized water and OMMT content on the UV-induced polymerization progress were systematically investigated. OMMT/PAA composite hydrogels with superior water absorbency and excellent mechanical properties can be obtained when the initial amount of deionized water is $95.7 \%$ (the ratio is $\mathrm{H}_{2} \mathrm{O} /\left(\mathrm{H}_{2} \mathrm{O}+\mathrm{AA}\right.$ ) in weight) and the content of OMMT is $10 \%$ (the ratio is OMMT/ (OMMT + AA) in weight). The maximum water absorbency in deionized water is $2786 \mathrm{~g} \mathrm{~g}^{-1}$ due to the highly reactive hydroxy groups in OMMT, which is more than 5 times higher than that of PAA hydrogels $\left(545 \mathrm{~g} \mathrm{~g}^{-1}\right)$. The OMMT/PAA containing 10\% OMMT has excellent elasticity and can be stretched up to $2200 \%$ for a fresh-prepared hydrogel. It can also sustain a load weight of $650 \mathrm{~g}$, and has excellent recoverability. The compressive stress-strain measurements also verify that the OMMT/PAA hydrogel containing 10\% OMMT has obviously improved mechanical properties compared to the others. The combination of high water absorbency, excellent elasticity mechanical properties, efficient recoverability and facile preparation provides a new way for fabricating high-performance hydrogel materials.

\section{Introduction}

Superabsorbent polymers (SAPs) $)^{\mathbf{1 - 5}}$ are lightly cross-linked three dimensional polymeric networks materials which absorb huge volumes of distilled water, and retain it even under large external pressure. With these superior performances, SAPs have been used in a variety of fields such as agriculture and horticulture, ${ }^{6}$ sanitary goods, ${ }^{7}$ waste-water treatment, ${ }^{8}$ biomedical areas $^{9}$ and so on. In 2015, the global SAP consumption increased to 2.3 million metric tons. The global demand for SAPs is increasing and will reach 3.48 million metric tons in $2020 .^{10}$ This large market evokes a strong desire to develop novel SAPs materials with higher water absorbency and excellent mechanical properties. In recent years, polysaccharide-based, ${ }^{\mathbf{1 1}}$ graphene oxide (GO)-based ${ }^{\mathbf{1 2}}$ and acrylic acid (AA)-based SAPs $^{13-25}$ have become ubiquitous and indispensable materials. Poly(acrylic acid) (PAA) hydrogels, in particular, dominate the market because of their low price and simple synthesis process. However, the weak absorbing capacities and poor mechanical properties of PAA hydrogels have severely restricted their commercial application. The ideal PAA-based SAPs

${ }^{a}$ College of Chemistry \& Chemical Engineering, Shaanxi Key Laboratory of Chemical Reaction Engineering, Yan'an University, Yan'an, Shaanxi 716000, P. R. China

${ }^{b}$ School of Chemistry and Environment, Beihang University, 100191, P. R. China. E-mail: henglp@iccas.ac.cn; Fax: +86-10-82627566 composite products should own not only high water absorbency but also excellent mechanical properties.

Presently, many efforts have been made to improve these performances of PAA-based hydrogels, such as exploiting composite hydrogels including inorganic clay materials, ${ }^{\mathbf{1 3 - 1 8}}$ graphene oxide, ${ }^{19,20}$ chitosan, ${ }^{21,22}$ silk sericin, ${ }^{23}$ maize bran, ${ }^{24}$ and designing double-network hydrogels. ${ }^{25}$ For example, Li et al. ${ }^{21}$ reported a dynamic cross-linked network via in situ polymerization of AA and acrylamide (AM) monomers in the aqueous solution of chitosan. The fracture strain of the obtained hydrogel increased to $1600 \%$, but the water absorbency has not been studied. Zhang ${ }^{24}$ synthesized maize bran-poly(acrylic acid) hydrogels with maximum water absorbency of $2507 \mathrm{~g} \mathrm{~g}^{-1}$, which is the highest water absorbing quantity of PAA-based hydrogels in the current literatures, but they did not refer to the mechanical properties. Compared with many work focusing on the synthesis and water absorbency capacity of PAA-based SAPs, there are fewer reports about mention of simultaneously owning high water absorbency and remarkable mechanical property. Zhong's group $^{25}$ prepared a single network structure PAA hydrogel with the water absorbency of $1800 \mathrm{~g} \mathrm{~g}^{-1}$ in deionized water and the fracture strain of $1952 \%$ by one-pot free radical polymerization to address this issue. However, this method needs complex synthesis procedure. Furthermore, for conventionally covalently cross-linked hydrogels, high water absorbency and good mechanical performance of polymer network are often inversely related. Therefore, it is a huge challenge to prepare SAPs 
hydrogels having high water absorbency and excellent mechanical properties simultaneously.

In this paper, we prepared organic montmorillonite (OMMT)/PAA SAPs composite hydrogels with high water absorbency and superior mechanical properties using simple UV irradiation polymerization. ${ }^{24,26}$ The influence of the initial amount of water in the precursor solution and OMMT contents in the composites on the performances of the prepared OMMT/ PAA composites hydrogels was systematically investigated. When the initial amount of deionized water was $95.7 \%$ and the content of OMMT was $10 \%$, the optimal OMMT/PAA SAPs hydrogels was obtained. The prepared hydrogels have a high water absorbing capacity of $2786 \mathrm{~g} \mathrm{~g}^{-1}$ in distilled water and exhibit excellent mechanical properties, in which the hydrogels can load the weight of $650 \mathrm{~g}$ and can be stretched to $2200 \%$ for a fresh-prepared hydrogel. The stress-strain measurement also verified that the introduction of OMMT into PAA hydrogel obviously improved the mechanical property of the hydrogel. Therefore, controlling the initial amount of water in polymerization and loading inorganic clay in the PAA network are the efficient approaches to improve the performances of PAA-based SAPs. The SAPs composites will have potential in waste-water treatment and biomedical fields.

\section{Experimental}

\subsection{Preparation of OMMT}

Montmorillonite (MMT) clay (LAPONITE® XLG, $\left[\mathrm{Mg}_{5.34} \mathrm{Li}_{0.66}\right.$ $\left.\mathrm{Si}_{8} \mathrm{O}_{20}(\mathrm{OH})_{4}\right] \mathrm{Na}_{0.66}$, diameter 200-300 nm, thickness 10-30 nm, Rockwood Ltd., UK) were used after treatment according to our previously reported method. ${ }^{26}$ MMT of $10 \mathrm{~g}$ dispersed in 1000 $\mathrm{mL}$ of deionized water followed by centrifugation to remove the large particle size MMT. Then, the obtained MMT (1.0 g) and cetyltrimethyl ammonium bromide (CTAB, $0.05 \mathrm{~g}$ ) were dispersed in $100 \mathrm{~mL}$ of deionized water and stirred at $80^{\circ} \mathrm{C}$ in a water bath for $3 \mathrm{~h}$. The resulting mixture was separated by Buchner funnel and washed with a large amount of deionized water, until $\mathrm{pH}=7$, to remove residual materials. After drying at $65{ }^{\circ} \mathrm{C}$ for $24 \mathrm{~h}$, the product was milled through a mortar and a white powder, CTAB-modified-MMT (OMMT), was obtained.

\subsection{Preparation of PAA and OMMT/PAA}

PAA hydrogels were fabricated via photopolymerization, in which 2,2-dimethoxy-2-phenylacetophenone (DMPA) and $\mathrm{N}, \mathrm{N}$ methylene diacrylamide (BIS) was used as photoinitiator and cross-linker, respectively. In detail, $0.05 \mathrm{~g}$ DMPA, $0.03 \mathrm{~g}$ BIS and $2.25 \mathrm{~g}$ AA monomer were added to a certain amount deionized water and then stirred at room temperature for $30 \mathrm{~min}$, followed by exposure to UV light $(365 \mathrm{~nm}, 40 \mathrm{~W})$ for $3 \mathrm{~h}$. The distance from UV lamp to sample was $5 \mathrm{~cm}$.

A certain amount of OMMT was completely dispersed in $95.7 \%$ of deionized water (the ratio of $\mathrm{H}_{2} \mathrm{O} /\left(\mathrm{H}_{2} \mathrm{O}+\mathrm{AA}\right.$ ) in weight) by stirring for $50 \mathrm{~min}$ to obtain the suspension solution of OMMT. Herein, the amount of OMMT was denoted by the ratio of OMMT/ (OMMT + AA) in weight. A transparent solution was prepared by blending $2.25 \mathrm{~g}$ AA, $0.05 \mathrm{~g}$ DMPA and $0.03 \mathrm{~g}$ BIS, followed by stirring at room temperature for $30 \mathrm{~min}$. Then the OMMT suspension solution was poured into the above-mentioned transparent solution and stirred at room temperature for $30 \mathrm{~min}$. The homogeneous mixture was exposed to a UV light with a wavelength of $365 \mathrm{~nm}$ for $3 \mathrm{~h}$, and the OMMT/PAA hydrogels were obtained.

\subsection{Measurement of water absorbency}

The water absorbency of the prepared hydrogels was measured via gravimetric analysis. The as-prepared hydrogel with a fixed weight was immersed in a large amount of deionized water at room temperature for different interval times $(2,4,7,10,12$ and 14 days). The swollen sample was filtered through an 80-mesh stainless screen to separate unabsorbed water. The equilibrium water absorbency of hydrogel $\left(Q: \mathrm{g} \mathrm{g}^{-1}\right)$ was calculated by using the following equation: $:^{27,28}$

$$
Q=\left(m_{2}-m_{1}\right) / m_{1}
$$

where, $m_{1}(\mathrm{~g})$ and $m_{2}(\mathrm{~g})$ are the weights of the sample in the dry state and the swollen state at a certain time, respectively. Water absorbency in salt solutions was tested in the same way. All of the experiments were performed in triplicate, and the results were averaged.

\subsection{Measurement of mechanical properties}

The tensile property of all the prepared hydrogels was measured by manual stretching. In detail, the initial hydrogel with the length of $4 \mathrm{~cm}$ was placed on the smooth surface and then stretched by hands at an average stretching velocity of $1 \mathrm{~mm}$ $\mathrm{s}^{-1}$. The length of the hydrogel after stretching was measured.

Compressive strength test was carried out by putting a weight with different mass on the prepared hydrogels. All hydrogels have a diameter about $1 \mathrm{~cm}$ and a thickness about $0.5 \mathrm{~cm}$. The hydrogels were loaded a weight with a certain mass for $5 \mathrm{~min}$, and then the weight was removed. Photographs of the hydrogel before and after loading weight were taken to record the morphology. The prepared hydrogels $(1 \mathrm{~cm} \times 1 \mathrm{~cm} \times 0.5$ $\mathrm{cm})$ were utilized to test the compressive stress-stain curves at room temperature.

\subsection{Characterization}

Photo-initiated polymerization was operated under a UV lamp (ZF-20D, China) with the light of $365 \mathrm{~nm}$. The photographs were taken on a digital camera (Pentax K-30, Japan). The structure of the as-prepared hydrogels was characterized by using a S-4800 field-emission scanning electron microscope (SEM, Hitachi, Japan), after sputtering the samples with a thin layer of gold. Thermal gravimetric analysis (TGA) was carried out by using the NETZSCH STA 449F3 (Germany) from 25 to $900{ }^{\circ} \mathrm{C}$ at a heating rate of $10{ }^{\circ} \mathrm{C} \mathrm{min}^{-1}$ under a flowing nitrogen atmosphere. IR Prestige-21 Fourier transform infrared spectrometer (Shimadzu, Japan) was used to record the vibration in functional groups of the samples (spectral range from 4000 to $500 \mathrm{~cm}^{-1}$ ). Stress-stain curves were measured in a universal mechanical testing machine (Instron 3365, USA). 


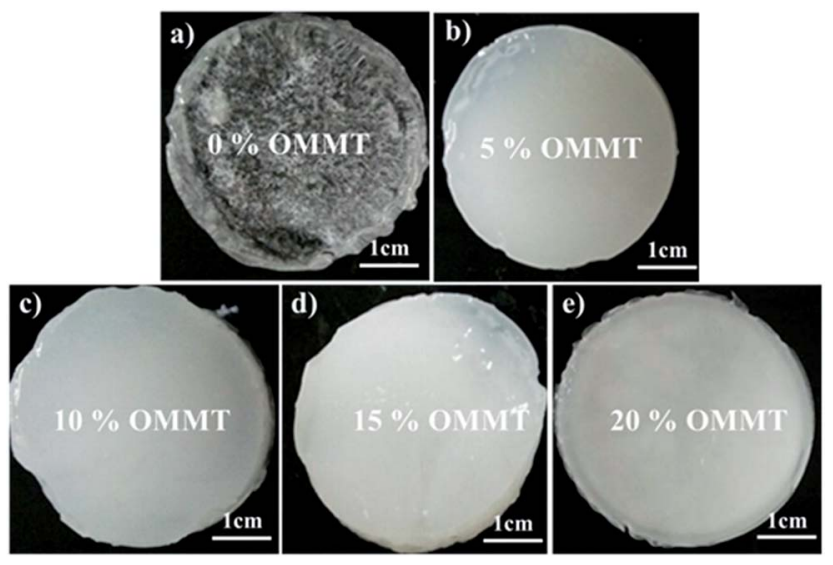

Fig. 1 The photographs of (a) PAA and $(b-e)$ OMMT/PAA hydrogels containing different amounts of OMMT.

\section{Results and discussion}

\subsection{Characterization of OMMT/PAA hydrogels}

The PAA hydrogel without introducing OMMT was transparent, as shown in Fig. 1a. Due to the low water absorbency and poor mechanical properties ${ }^{21}$ in many instances, hydrophilic MMT with unique physical and chemical properties, is expected to introduce into the hydrogels to improve their water absorb capacity and mechanical properties. The natural MMT clays, however, lack affinity with organic polymer because of their hydrophilicity. To render MMT more organophilic, MMT is usually treated with organic long alkyl chain ammonium salt (such as CTAB) before the preparation of MMT/polymer hydrogels. ${ }^{26}$ Here the MMT treated by CTAB was added to the precursor solution containing AA, DMPA and BIS, followed by UV radiation polymerization. By adjusting the amounts of OMMT, we fabricated various OMMT/PAA SAPs composite hydrogels, as shown in Fig. 1b-e. The samples present a milk color, which is obviously different from the pure PAA hydrogel.

SEM images, as exhibited in Fig. 2, were employed to characterize the structure of PAA and OMMT/PAA hydrogels. For PAA hydrogel (Fig. 2a), three-dimensional network macroporous structure can be observed obviously. The macropores were constructed from smooth PAA sheet and the diameters were ranged from $1.71 \mu \mathrm{m}$ to $15.7 \mu \mathrm{m}$. The OMMT/PAA hydrogels (Fig. 2b) remained the three-dimensional network macroporous structure, while the pore diameters became smaller (1.0
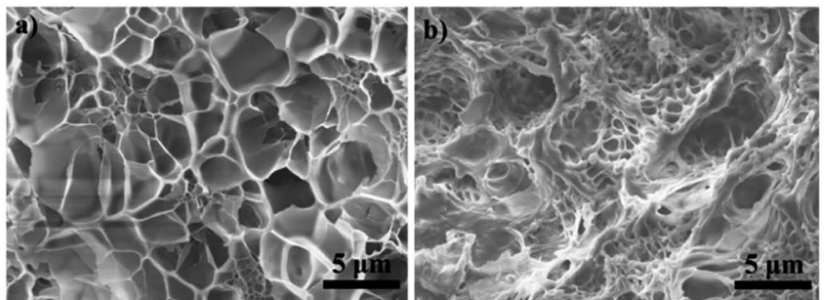

Fig. 2 SEM images of (a) PAA prepared in $95.7 \%$ of deionized water and (b) OMMT/PAA containing 10\% OMMT. The scale bar is $5 \mu \mathrm{m}$. $\mu \mathrm{m}$ to $4.36 \mu \mathrm{m})$, which is beneficial to increase the special surface area of the hydrogels. In addition, many pores appeared in the initial smooth PAA sheet and the pore wall became rough. The OMMT liked as glue which bonded the macropores together, which is conducive to improve the mechanical properties of hydrogels. The results demonstrate that the structure of hydrogel is significantly affected by the OMMT.

Fig. 3a shows the IR spectra of MMT, OMMT, PAA and OMMT/ PAA. For MMT, the characteristic peaks at 3633 and $3435 \mathrm{~cm}^{-1}$ can be attributed to the stretching vibration of hydroxyl $(-\mathrm{OH})$ on the surface layers of MMT and the interlayer water of MMT. The peaks at 1645 and $910 \mathrm{~cm}^{-1}$ derive from the bending vibration of $-\mathrm{OH}$, while the absorptions at 1041 and $796 \mathrm{~cm}^{-1}$ can be ascribed to the stretching vibration of $\mathrm{Si}-\mathrm{O}-\mathrm{Si}^{26-28}$ New characteristic absorption peaks at 2929, 2852 and $1478 \mathrm{~cm}^{-1}$, arisen from the vibration of saturated $\mathrm{C}-\mathrm{H}$, can be obviously observed in the spectrum of OMMT after treating MMT with CTAB, demonstrating the CTAB had intercalated into the layers of MMT. ${ }^{26}$ The characteristic absorptions of hydroxyl $(-\mathrm{OH})$ and carbonyl $(-\mathrm{C}=\mathrm{O})$ in carboxyl group $(-\mathrm{COOH})$ of PAA occur at 3435 and $1718 \mathrm{~cm}^{-1}$, respectively. In the spectrum of OMMT/PAA, the absorption peaks at 3426 and $1706 \mathrm{~cm}^{-1}$ testify the presence of associated $-\mathrm{COOH}$, and the peaks at 2992, 1039 and $796 \mathrm{~cm}^{-1}$, represent the saturated $\mathrm{C}-\mathrm{H}$ and $\mathrm{Si}-\mathrm{O}$, respectively. The absorption bands observed in IR spectra confirmed that OMMT were grafted onto PAA threedimensional polymeric network, and the clays also participated in polymerization reaction, which indicated that OMMT had been intercalated into PAA. The interaction between OMMT and PAA is probably ascribed to hydrogen bonding between hydroxyl groups on PAA and the $\mathrm{Si}-\mathrm{OH}$ or $\mathrm{Si}-\mathrm{O}$ groups on the OMMT surface.

Thermal gravimetric analysis (TGA) ${ }^{26-29}$ was carried out to investigate the amount of OMMT intercalated into PAA. The TGA curves of PAA hydrogel and the OMMT/PAA hydrogels are presented in Fig. 3b. All the TGA curves exhibit three thermal decomposition stages. At the initial stage, all the hydrogels show a loss of approximately $17-20 \mathrm{wt} \%$ as the temperature increased to $221{ }^{\circ} \mathrm{C}$, which is due to the presence of moisture in the samples. There is a sharp weight loss for each hydrogel from 221 to $324^{\circ} \mathrm{C}$, in the second decomposition stage, derived from the elimination of water molecules from the neighboring carboxy of PAA polymer chains. The complete decomposition of PAA hydrogel occurred in the last stage when temperature ranged from 324 to $481{ }^{\circ} \mathrm{C}$. For OMMT/PAA composite hydrogels
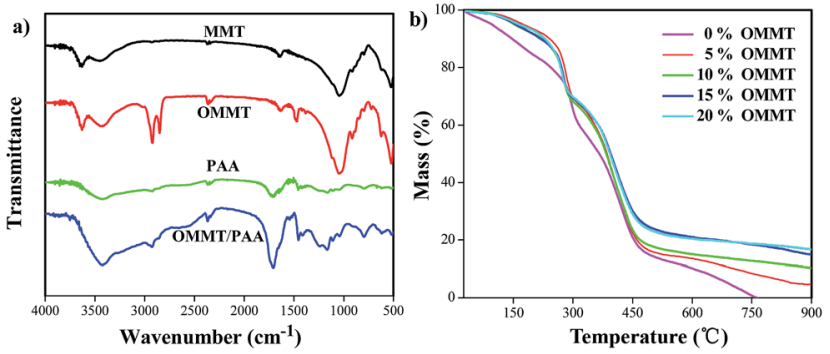

Fig. 3 (a) FTIR spectra of MMT, OMMT, PAA and OMMT/PAA prepared from 10\% OMMT; (b) TGA curves of the dried PAA and OMMT/PAA hydrogels containing different amounts of OMMT. 
containing different amounts of OMMT (5\%, 10\%, 15\% and $20 \%$ ), the mass loss are $95.4 \%, 89.67 \%, 85.1 \%$, and $83.3 \%$, respectively, at the final stage, which demonstrates that the amount of the residue are nearly consistent with the addition amount of OMMT. In addition, the TGA curves also show that the thermal stability of the OMMT/PAA SAPs composites is higher than that of PAA hydrogel. The OMMT/PAA hydrogels presented a much higher thermal stability up to $900{ }^{\circ} \mathrm{C}$ with only less weight lost, while PAA hydrogel decomposed completely at $750{ }^{\circ} \mathrm{C}$. This clearly indicated that the thermal stability of OMMT/PAA SAPs can be significantly improved by introducing OMMT clay, which can act as a heat barrier material into the polymeric network.

\subsection{Water absorbency of OMMT/PAA hydrogels}

We firstly investigated the influence of water content in the precursor solution by varying the deionized water amounts from $68.9 \%$ to $96.9 \%$ while keeping the other variables constant. So we can find out the optimal water amount for preparing the PAA hydrogel with the maximum water absorbency. The PAA hydrogels fabricated from different water contents were immersed in deionized water for different days and the averaged water absorbency were obtained, as shown in Fig. 4a. As increasing the immersion time, there were not obvious changes for water absorbency when the water content in the precursor solution was lower than $89.9 \%$. The water absorbency increased with prolonging the immersion time when the water content changed from $93.0 \%$ to $95.7 \%$. However, the water absorbency decreased with increasing the soak time when the water content in the precursor solution was higher than $96.4 \%$. In addition, PAA hydrogel could not be obtained when the water amount in the precursor solution reached $97.3 \%$. From Fig. 4 a, we also can see that the water absorbency obtained from the water content of $95.7 \%$ in the precursor solution was obviously higher than those obtained from the other water contents when the immersion time was same (here the time was more than 4 days). Fig. $4 \mathrm{~b}$ exhibits the water absorbency in deionized water measured at 14 days for different PAA hydrogels prepared from various water contents. The results demonstrate that the alteration of water content has a significantly effect on the water absorbance capacity for PAA hydrogels. The reason is that the water content in precursor solution directly influenced the polymerization of monomer and the cross-linking density on per unit because of invariable contents of DMPA, BIS and AA. As the water content changed from $68.9 \%$ to $95.7 \%$, the water absorbency increased because more water provided more hydrophilic hydroxyl groups attached onto the polymeric backbone. ${ }^{6}$ A sharp decrease could be observed when the water content was over $95.7 \%$ due to the increased space between the polymeric networks induced by low cross-linked density of PAA hydrogel. Therefore, the degree of cross-linking needs to be balanced to prepare PAA hydrogels with adequate absorption capacity and ideal mechanical properties. The maximum water absorbency of $545 \mathrm{~g} \mathrm{~g}^{-1}$ could be obtained when the water content in the precursor solution was $95.7 \%$. Therefore, $95.7 \%$ of the water content was utilized for the following fabrication of OMMT/PAA hydrogels.
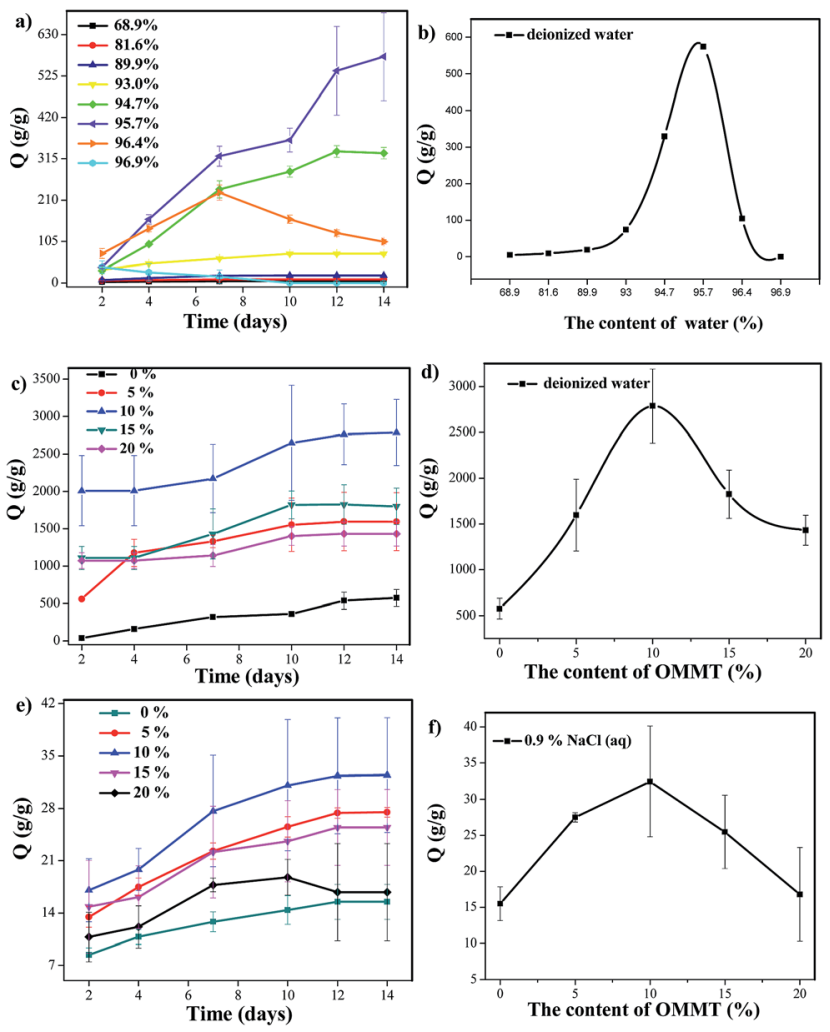

Fig. 4 (a) The water absorbency of PAA hydrogels prepared from different water contents in the precursor solution when immersed in deionized water for different days and (b) the water absorbency measured at 14 days. (c-f) Effect of OMMT contents on water absorbency of OMMT/PAA hydrogels, prepared from the precursor solution where the initial water content was $95.7 \%$ in deionized water (c and d) and in $0.9 \mathrm{wt} \% \mathrm{NaCl}$ aqueous solution (e and f).

The various composite OMMT/PAA hydrogels were fabricated by adjusting the OMMT content during the process of photopolymerization, in which the amounts of AA $(2.25 \mathrm{~g})$, DMPA (0.05 g), BIS (0.03 g), and deionized water (95.7\%) were constant. Their water absorbency in deionized water and 0.9 $\mathrm{wt} \% \mathrm{NaCl}$ aqueous solution was investigated, as shown in Fig. 4c-f. From Fig. 4c and e, we can see an obvious trend that the water absorbency of all the prepared hydrogels increased at the initial stage, and then leveled off until it reached a platform within $\sim 12$ days. As the swelling continued, more water diffused into the network and gradually weakened the osmotic pressure difference. As a result of continuously overcoming the osmotic pressure inside the hydrogels, the swelling ability finally reached equilibrium. ${ }^{14}$ Fig. $4 \mathrm{~d}$ exhibits that the maximum water absorbency of OMMT/PAA hydrogels reached $2786 \mathrm{~g} \mathrm{~g}^{-1}$ in deionized water when the content of OMMT was $10 \%$. The obtained water absorbency is more than 5 times higher than that of PAA hydrogels (545 $\mathrm{g} \mathrm{g}^{-1}$ ), and it is also higher than those reported in the literatures. ${ }^{23,25}$ The high water absorbency of OMMT/PAA can be attributed to the highly reactive - $\mathrm{OH}$ groups in OMMT which bond water molecules. Thereafter, a sharp decrease of the water absorbency could be observed clearly when the amount of OMMT was more than 10\%. Actually, the 
-Si-OH groups in OMMT can also chemically participate in the polymerization reaction and generate additional network points to enhance the OMMT/PAA composite hydrogels' crosslinking density. The more OMMT, beyond the equilibrium, may cause a hindrance to the OMMT/PAA hydrogels' polymeric network elasticity, which may reduce the water absorption efficiency. ${ }^{\mathbf{1 4}}$ Furthermore, the high amount of OMMT above the equilibrium value may interrupt the graft copolymerization of AA into OMMT layer, thus resulting in decreased water absorbency. ${ }^{\mathbf{1 6}}$ The present experimental results are also in good agreement with our previous study. ${ }^{26}$ Therefore, a suitable amount of inorganic clay is needed to prepare an ideal SAPs with both improved swelling and mechanical properties. The water absorption behavior of OMMT/PAA composite hydrogels in 0.9 wt\% NaCl solution (Fig. 4e and f) is similar to that measured in deionized water and the maximum water absorbency is $32 \mathrm{~g} \mathrm{~g}^{-\mathbf{1}}$.

\subsection{Mechanical property of OMMT/PAA hydrogels}

The tensile properties of all the as-prepared hydrogels are shown as plots of strain in Fig. 5. Fig. 5a shows the photographs of the initial prepared PAA and OMMT/PAA hydrogels. ${ }^{21,25,30} \mathrm{We}$ can see clearly that all the hydrogels are free-standing and retain their shape and dimensions. The hydrogels can easily stretch like an elastomer, as shown in Fig. 5b-f. The simple stretching experiments were performed by hands, in which the average stretching velocity was about $1 \mathrm{~mm} \mathrm{~s}^{-1}$. For PAA hydrogel, it can be stretched from $1 \mathrm{~cm}$ of the initial length to $7 \mathrm{~cm}$ and the tensile distance is $6 \mathrm{~cm}$ (Fig. 5b). After introducing different amounts of OMMT (5\%, 10\%, 15\%, 20\%), the tensile distances are $12 \mathrm{~cm}, 22 \mathrm{~cm}, 15 \mathrm{~cm}$, and $12 \mathrm{~cm}$ (Fig. 5c-f), respectively, which are obviously superior to PAA hydrogel. Noteworthily, the OMMT/PAA hydrogel with 10\% OMMT is so extensible that it can be stretched from $1 \mathrm{~cm}$ to $23 \mathrm{~cm}$ (Fig. $5 \mathrm{~d}$ ), which means the stretching can be done up to $2200 \%$ strain and

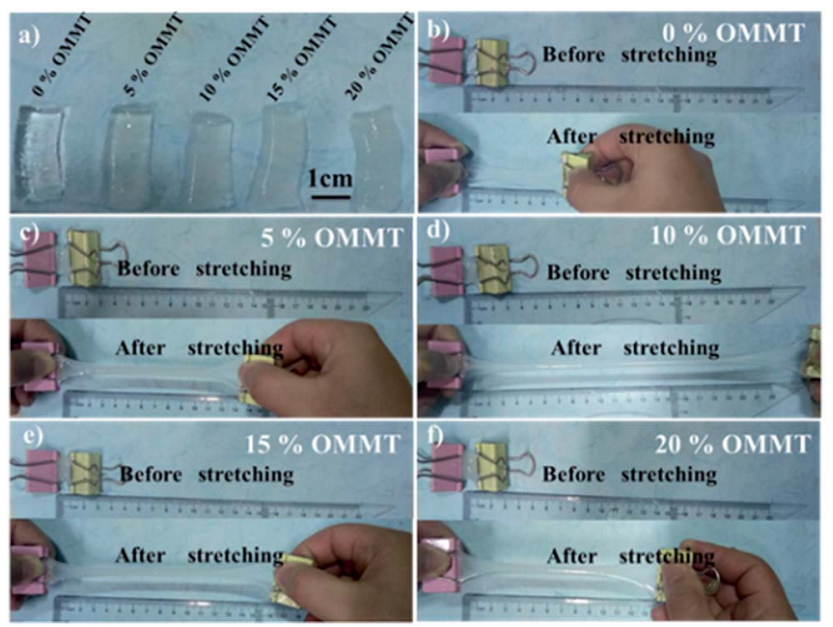

Fig. 5 (a) Photographs of the initial morphology of all the prepared hydrogels. $(b-f)$ Photographs of the hydrogels containing different amounts of OMMT before (the top in each photo) and after stretching (the bottom in each photo). The products can recover to their original shape immediately after removing the stretching. The average stretching velocity is about $1 \mathrm{~mm} \mathrm{~s}^{-1}$. the product can be stretched to many times of the original length. Meanwhile, the OMMT/PAA hydrogels exhibited no residual strain after continuous deformation-resting processes, which again reveals their elastic character and demonstrates their high extensibility. The stretchability of OMMT/PAA hydrogels became weaker when OMMT content is too high or too low because more inorganic rigid OMMT will make the hydrogels more fragile, while fewer OMMT will affect the gel strength. The results testified that the elasticity of the hydrogels is largely influenced by the content of OMMT. ${ }^{14}$

Compressive strength test was also carried out by putting a weight on the as-prepared hydrogels. The initially prepared PAA and OMMT/PAA hydrogels containing different amounts of OMMT (Fig. 6a) were loaded a weight (Fig. 6b) and then removing it. ${ }^{19}$ Fig. 6 shows the photographs of PAA and different OMMT/PAA hydrogels after removing the weight of $100 \mathrm{~g}$. Obviously, the pure PAA hydrogel was very brittle and easily destroyed under the compressive loading of $100 \mathrm{~g}$, while the OMMT/PAA composite hydrogels kept their initial shape perfectly. It testifies that OMMT can effectively enhance the gel strength and improve the mechanical property of the composite network, which is due to its inorganic rigidness. When the loading weight was increased to $650 \mathrm{~g}$, only the sample containing $10 \%$ OMMT could maintain the initial morphology and could not be crushed. The compressive performance of the OMMT/PAA composites with 10\% OMMT exhibited a significant reinforcement compared to other hydrogels. This reinforcement is mainly attributed to both the unique mechanical nature of OMMT and the intermolecular interactions between OMMT and the polymer chains, which is beneficial to the effective load transfer in the hydrogel networks and lead to the enhanced load-bearing ability. ${ }^{13}$ The results demonstrate that the suitable amount of OMMT plays a key role for fabricating the hydrogels with excellent mechanical properties. ${ }^{31}$ It is noted that the OMMT/PAA hydrogels can recover to their original shape

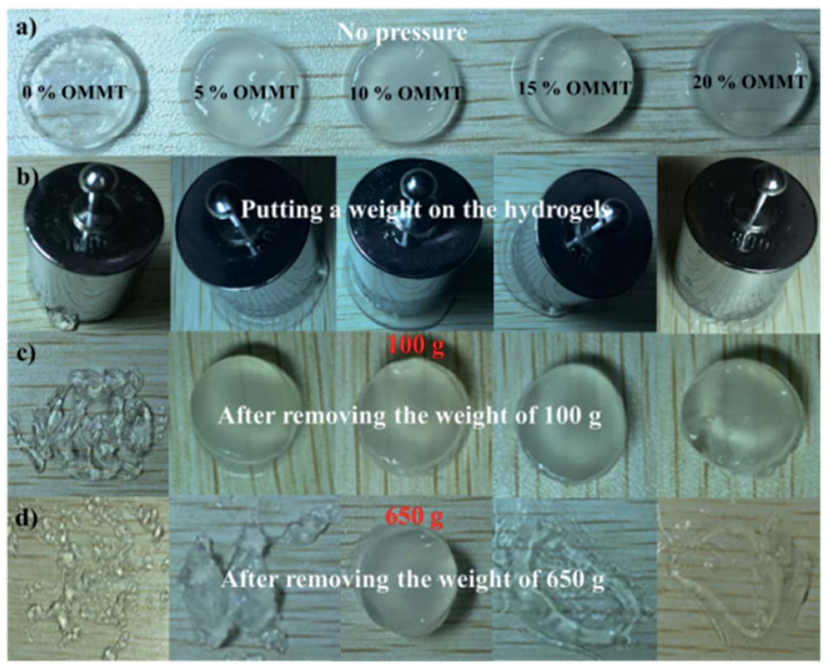

Fig. 6 Photographs of PAA and OMMT/PAA hydrogels containing different amounts of OMMT before (a), during (b) and after (c and d) loading a certain external pressure. The weights of load are $100 \mathrm{~g}$ and $650 \mathrm{~g}$, respectively. 


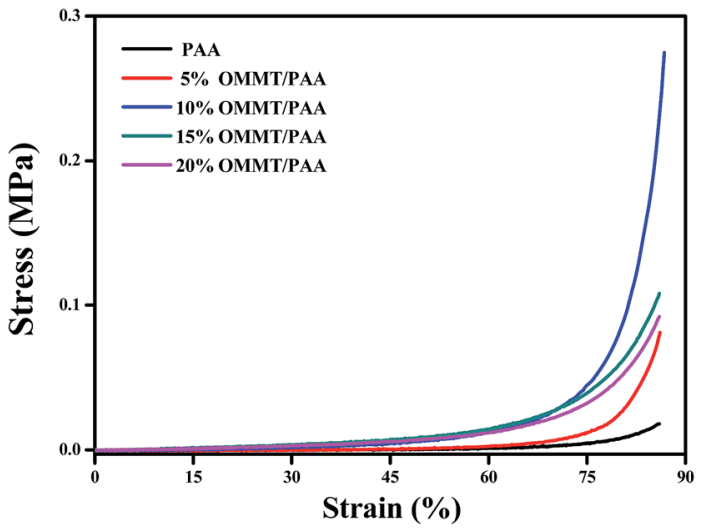

Fig. 7 Compressive stress-strain of PAA and OMMT/PAA hydrogels with different amounts of OMMT.

immediately after removing the weight. In fact, a similar mechanical reinforcement has also been evidenced in other OMMT/polymer composite hydrogel systems. ${ }^{14}$ Therefore, the addition of OMMT not only shows crucial effects on the swelling behavior in deionized water, but also can evidently enhance the mechanical properties of composite hydrogels.

Compressive stress-strain measurement ${ }^{32,33}$ was also carried out to verify the mechanical properties of the as-prepared hydrogels. Fig. 7 exhibits the stress-strain curves for the hydrogels under $86 \%$ compression. The OMMT/PAA hydrogels exhibited improved mechanical properties compared to PAA hydrogel. During $86 \%$ compression strain, the OMMT/PAA hydrogels containing different amounts of OMMT displayed different stress. The stress is $0.018,0.081,0.275,0.108$ and $0.092 \mathrm{MPa}$, respectively, for PAA and different OMMT/PAA (5\%, $10 \%, 15 \%$, and $20 \%$ ) hydrogels. The OMMT/PAA hydrogel containing $10 \%$ OMMT, particularly, exhibited an obviously higher stress than PAA hydrogel and other OMMT/PAA hydrogels, which means it has a better mechanical property than others. The PAA hydrogels without OMMT clay easily fractured under $86 \%$ compression. In contrast, OMMT/PAA containing $10 \%$ OMMT can endure high stress and quickly recover their original shape after the loading force is released, which demonstrated the excellent mechanical properties of the prepared OMMT/PAA hydrogels. Further tensile measurements of the hydrogels were tried by us, but the hydrogel is too fragile to be applied in our tensile experiments. So we cannot obtain the tensile curve.

\section{Conclusions}

The OMMT/PAA SAPs composite hydrogels were synthesized by UV irradiation polymerization of AA monomer, using BIS as a cross-linker, DMPA as an initiator and OMMT as inorganic filler. By adjusting the amounts of OMMT in the OMMT/PAA composite hydrogels, the maximum water absorbency of $2786 \mathrm{~g} \mathrm{~g}^{-1}$ in deionized water was obtained when the OMMT content was $10 \%$. The mechanical properties of the OMMT/PAA hydrogels are significantly influenced by the contents of OMMT. The OMMT/PAA containing 10\% OMMT has excellent elasticity and can be stretched up to $2200 \%$ strain. It can also sustain a load weight of $650 \mathrm{~g}$, and recover to its initial shape perfectly. The stress-strain measurement indicated that the elasticity of OMMT/PAA hydrogel is superior to PAA hydrogel. The asprepared OMMT/PAA composite hydrogels presented a remarkable improvement both in water absorbency and mechanical properties compared with those of the corresponding PAA hydrogels. The combination of high water absorbency, excellent mechanical properties and efficient recoverability endows the OMMT/PAA hydrogels with wide applications in wastewater treatment or biomedical engineering.

\section{Acknowledgements}

This work was supported by the National Natural Science Foundation (21663032, 51541301), the Natural Science Fundamental Research Program Key Projects of Shaanxi Province (2016JZ005), the Key Laboratory Research Program of the Education Department of Shaanxi Provincial Government (15JS121) and the National Research Fund for Fundamental Key Projects (2014CB931802).

\section{References}

1 J. F. Mukerabigwi, S. J. Lei, L. Fan, H. L. Wang, S. Y. Luo, X. Y. Ma, J. Qin, X. Y. Huang and Y. Cao, RSC Adv., 2016, 6, 31607-31618.

2 Y. L. Ma, Y. J. Sun, Y. J. Fu, G. Z. Fang, X. R. Yan and Z. H. Guo, Chemosphere, 2016, 163, 610-619.

3 P. C. Zhang, F. L. Zhang, C. Q. Zhao, S. T. Wang, M. J. Liu and L. Jiang, Angew. Chem., Int. Ed., 2016, 55, 3615-3619.

4 A. Serafim, C. Tucureanu, D. G. Petre, D. M. Dragusin, A. Salageanu, S. V. Vlierberghe, P. Dubruelc and I. C. Stancu, New J. Chem., 2014, 38, 3112-3126.

5 X. N. Shi, W. B. Wang, Y. A. Zheng and A. Q. Wang, RSC Adv., 2014, 4, 50478-50485.

6 X. Y. Liang, Y. Zhang, L. Liu and J. M. Yao, BioResources, 2013, 8, 130-144.

7 G. R. Bardajee, A. Pourjavadi, R. Soleyman and S. Ghavami, Adv. Polym. Technol., 2012, 31, 41-45.

8 L. C. Davies, J. M. Novais and S. Martins-Dias, Bioresour. Technol., 2004, 95, 259-268.

9 D. J. Feng, Ind. Eng. Chem. Res., 2014, 53, 12760-12769.

10 Global and China Superabsorbent Polymers (SAPs) Industry Report, 2016-2020, published by Research In China, 2016.

11 X. P. Shen, J. L. Shamshina, P. Berton, G. Gurau and R. D. Rogers, Green Chem., 2016, 18, 53-75.

12 Z. Q. Zhu, H. X. Sun, X. J. Qin, L. Jiang, C. J. Pei, L. Wang, Y. Q. Zeng, S. H. Wen, P. Q. La, A. Li and W. Q. Deng, J. Mater. Chem., 2012, 22, 4811-4817.

13 Y. J. Lin, F. C. Fsu, C. W. Chou, T. H. Wu and H. R. Lin, J. Mater. Chem. B, 2014, 2, 8329-8337.

14 G. B. Marandi, G. R. Mahdavinia and S. Ghafary, J. Polym. Res., 2011, 18, 1487-1499.

15 M. A. Fareed and A. Stamboulis, J. Mater. Sci.: Mater. Med., 2014, 25, 91-99. 
16 J. Z. Gao, D. L. Ma, Q. F. Lu, Y. Li, X. F. Li and W. Yang, Plasma Chem. Plasma Process., 2010, 30, 873-883.

17 L. Z. Zhao, C. H. Zhou, J. Wang, D. S. Tong, W. H. Yu and H. Wang, Soft Matter, 2015, 11, 9229-9246.

18 T. Q. Guo, M. C. Li, L. P. Heng and L. Jiang, $R S C A d v .$, 2015, 5, 62078-62083.

19 Y. W. Huang, M. Zeng, Z. J. Feng, D. Yin, Q. Y. Xu and L. R. Fan, $R S C A d v$., 2016, 6, 3561-3570.

20 Y. Yu, L. C. X. D. Andrade, L. M. Fang, J. Ma, W. J. Zhang and Y. H. Tang, J. Mater. Sci., 2015, 50, 3457-3466.

21 J. Li, Z. L. Su, X. D. Ma, H. J. Xu, Z. X. Shi, J. Yin and X. S. Jiang, Mater. Chem. Front., 2017, DOI: 10.1039/ c6qm00002a.

22 J. You, S. Y. Xie, J. F. Cao, H. Ge, M. Xu, L. N. Zhang and J. P. Zhou, Macromolecules, 2016, 49, 1049-1059.

23 X. B. Hu, Polym. Bull., 2011, 66, 447-462.

24 M. Y. Zhang, Z. Q. Cheng, T. Q. Zhao, M. Z. Liu, M. J. Hu and J. F. Li, J. Agric. Food Chem., 2014, 62, 8867-8874.
25 M. Zhong, Y. T. Liu, X. Y. Liu, F. K. Shi, L. Q. Zhang, M. F. Zhu and X. M. Xie, Soft Matter, 2016, 12, 5420-5428.

26 L. P. Heng, T. Q. Guo, B. Wang, Y. Q. Zhang and L. Jiang, RSC Adv., 2015, 5, 102378-102383.

27 J. F. Mukerabigwi, S. J. Lei, H. L. Wang, S. Y. Luo, X. Y. Ma, J. Qin, X. Y. Huang and Y. Cao, RSC Adv., 2015, 5, 8373283742.

28 J. D. Gao, Q. Yang, F. T. Ran, G. F. Ma and Z. Q. Lei, Appl. Clay Sci., 2016, 132-133, 739-747.

29 Y. H. Zhang, Q. F. Gu, J. Yin, Z. G. Wang and P. X. He, Adv. Polym. Technol., 2014, 33, 21400-21407.

30 M. Zhong, Y. T. Liu and X. M. Xue, J. Mater. Chem. B, 2015, 3, 4001-4008.

31 Z. Y. Li, Y. L. Su, B. Q. Xie, X. G. Liu, X. Gao and D. J. Wang, J. Mater. Chem. B, 2015, 3, 1769-1778.

32 L. Lin, M. J. Liu, L. Chen, P. P. Chen, J. Ma, D. Han and L. Jiang, Adv. Mater., 2010, 22, 4826-4830.

33 H. Li, D. Z. Hao, J. B. Fan, S. F. Song, X. L. Guo, W. L. Song, M. J. Liu and L. Jiang, J. Mater. Chem. B, 2016, 4, 4662-4666. 